\title{
The axiverse induced dark radiation problem
}

\author{
Bobby Acharya $^{a, b}$ and Chakrit Pongkitivanichkul ${ }^{a}$ \\ ${ }^{a}$ Theoretical Particle Physics $\&$ Cosmology Group, Department of Physics, King's College London, \\ Strand, London, WC2R 2LS, U.K. \\ ${ }^{b}$ The Abdus Salam International Centre for Theoretical Physics, \\ Strada Costiera 11, Trieste, Italy \\ E-mail: bobby.acharya@kcl.ac.uk, chakrit.pongkitivanichkul@kcl.ac.uk
}

Abstract: The string/ $M$ theory Axiverse - a plethora of very light Axion Like Particles (ALPs) with a vast range of masses - is arguably a generic prediction of string/ $M$ theory. String/ $M$ theory also tends to predict that the early Universe is dominated by moduli fields. When the heavy moduli decay, before nucleosynthesis, they produce dark radiation in the form of relativistic ALPs. Generically one estimates that the number of relativistic species grows with the number of axions in the Axiverse, in contradiction to the observations that $N_{\text {eff }} \leq 4$. We explain this problem in detail and suggest some possible solutions to it. The simplest solution requires that the lightest modulus decays only into its own axion superpartner plus Standard Model particles and this severely constrains the moduli Kahler potential and mass matrix.

KEYWORDS: Strings and branes phenomenology

ARXIV EPRINT: 1512.07907 


\section{Contents}

1 Introduction 1

2 The axiverse induced dark radiation problem 3

3 String/ $M$ theory examples $\quad 7$

$\begin{array}{lll}3.1 & \text { Calabi-Yau compactifications } & 7\end{array}$

3.2 Diagonal Kahler metrics 9

3.2.1 Mass matrix in $G_{2}$ compactified M-theory 10

4 Conclusions and outlook $\quad 12$

$\begin{array}{ll}\text { A Decay coefficients } & 13\end{array}$

\section{Introduction}

String/ $M$ theory is a theoretical framework which predicts that there are six or seven extra dimensions of space. Even though the energy scale of the extra dimensions might be large, e.g. at the GUT scale, their lowest energy excitations, the moduli fields, typically have a much smaller mass, such as the supersymmetry breaking scale. These moduli fields appear in the low energy effective description of physics as scalar fields which couple to matter through higher dimension operators suppressed by the Planck scale, $m_{p l}$. This is natural since the moduli are, in fact, extra dimensional gravitons. In the string/ $M$ theory description of physics, the value of all Standard Model couplings and masses become the vacuum expectation values of the moduli fields. As an example, the Maxwell term in the Lagrangian, $L=\frac{1}{4 e^{2}} F_{\mu \nu}^{2}$ becomes of the form $\frac{s}{m_{p l}} F_{\mu \nu}^{2}$ for a particular modulus field $s$. The expectation values of the moduli fields are interpreted as determining the size and shape of the extra dimensions and the fact that most Standard Model couplings are weak implies that the extra dimensions are moderately large compared to the fundamental string/ $M$ theory scale, which further implies we can usually use a supergravity Lagrangian to describe the low energy physics.

String/ $M$ theory also predicts the existence of axion like particles (ALPs) - these are periodic, pseudo-scalars which arise as zero modes of the antisymmetric tensor fields present in all low energy descriptions of string/ $M$ theory $[1,2]$. In fact, in models with low energy supersymmetry, which is what we will assume from here onwards, the axions and moduli usually pair up to form the complex scalar fields which appear in the chiral supermultiplets in four dimensional supergravity theories. For example, related to the Maxwell term above will be a term like $\frac{a}{f_{a}} F_{\mu \nu} \tilde{F}^{\mu \nu}$ where $a$ is an axion field and $f_{a}$ is the "axion decay constant", which is typically of order the compactification scale, and often of 
order $M_{G U T}$. With this logic: for every U(1) and every simple factor in the full gauge group we will have one modulus field and one axion. Similarly, the magnitudes and phases of the entries of the Standard Model Yukawa matrices are respectively related to the moduli and axion vevs. Continuing in this fashion and including supersymmetric couplings as well, we see that the full theory could have hundreds, if not more, moduli and axions.

Since the axions arise as zero modes of antisymmetric gauge fields, the number of axions is determined by the number of harmonic antisymmetric tensor fields on the extra dimensions. This number is a topological invariant of the extra dimensions (a Betti number), so if the extra dimensions have a suitably rich topology there will be a large number of axions present in the spectrum. One then assumes that "rich topologies" are generic since simple topologies are rare. This was the argument given in [3] leading to the notion of the string/ $M$ theory Axiverse. This is clearly consistent with the argument given above based simply upon couplings. Note that specific examples of string/ $M$ theory compactifications could, and do, exist with very few light axions in the spectrum. In these examples, the topology is relatively simple and, equivalently, the axions you would expect based on the couplings argument have obtained a large mass through a direct breaking of the axion shift symmetries by the background geometry.

Extremely weakly coupled scalar fields like moduli and axions can have a considerable impact on cosmological dynamics due to the "vacuum misalignment" mechanism [4-6]. At very early times when the Hubble scale $H$ is above the masses of these particles, the fields are frozen at order one values ( $m_{p l}$ for the moduli and $f_{a}$ for the axions). Then, as the Universe expands and $H$ decreases, when $H$ becomes of order $m_{s}$ or $m_{a}$, the equation of motion requires the field to start oscillating around the minimum with a frequency of order $m_{s}$ or $m_{a}$. Since the corresponding contribution to the energy density will dilute like matter, even if the Universe was radiation dominated prior to this point, a modulus field will quickly dominate the Universe since its energy density is comparable to radiation at the onset of the oscillations. Next, when the Hubble scale reduces to be of order the modulus decay width, $\Gamma_{s} \sim \frac{m_{s}^{3}}{m_{p l}^{2}}$, the modulus field decays. This happens during nucleosynthesis for $m_{s} \sim \mathrm{TeV}$, which is known as the cosmological moduli problem [7, 8]. This problem will be avoided if $m_{s} \geq 30 \mathrm{TeV}$; one could also avoid it by assuming that the Hubble scale after inflation is always smaller than $m_{s}$ or if there is a late period of inflation which dilutes the moduli fields, however, both of these options require tuning and are presumably not generic. Therefore, we conclude that string/M theory seems to predict that the early Universe prior to nucleosynthesis is matter dominated.

The axions also participate in the vacuum misalignment mechanism but there are important differences. The shift symmetries that these fields enjoy protects their masses from perturbative contributions, hence they receive masses only from non-perturbative effects, such as instantons, which at weak coupling are exponentially small. The resulting very small axion masses means that the axion lifetimes are, unlike the moduli, generically extremely long, with lifetimes that can easily be cosmologically relevant. Hence, there will be a contribution to the energy density in the form of axion fields today, which behaves as cold dark matter. Notice that the decay of the moduli releases a large amount of entropy 
which dilutes any relics which existed prior to nucleosynthesis, e.g. ten orders of magnitude dilution is typical. This significantly weakens the upper bound on the QCD axion decay constant, compared to radiation dominated Universes, to be of order $10^{15} \mathrm{GeV}$ [9-12]. This effect also significantly dilutes other relics that may have formed previously, such as domain walls, monopoles or thermal WIMPs.

For our present study, the key point is that the moduli can decay into Standard Model particles, into supersymmetric particles as well as axions. Since the axions are so light and the moduli have masses in the tens of $\mathrm{TeV}$ regime, axions produced this way will be relativistic with energies of order several $\mathrm{TeV}$. The expansion of the Universe and precision cosmological observables are sensitive to the relative abundance of relativistic particles. This can be captured by the observable called $N_{\text {eff }}$ which is "the effective number of neutrino species", but is actually sensitive to all forms of relativistic matter, regardless of how such matter couples to the Standard Model. In that sense, $N_{\text {eff }}$ provides a very useful probe of additional, "hidden," sectors beyond the Standard Model. The Standard Model prediction for $N_{\text {eff }}$ at the time of recombination is 3.045, whilst measurements from CMB observations by WMAP 9-year polarisation data [13], South Pole Telescope [14], Atacama Cosmology Telescope [15], and Planck 2015 [16] are $N_{\text {eff }}=3.84 \pm 0.40$ (WMAP9), $N_{\text {eff }}=3.62 \pm 0.48$ (SPT), $N_{\text {eff }}=2.79 \pm 0.56(\mathrm{ACT}), N_{\text {eff }}=3.15 \pm 0.23$ (Planck2015) respectively. In a sense, this is a surprising result since one might expect $N_{\text {eff }}$ to be much, much larger naively.

So, from the perspective of string/ $M$ theory or the idea of "hidden sectors" more generally, the question actually becomes: why is $N_{\text {eff }}$ so small? For instance, if, as we have already argued, there are large numbers of light axions and the moduli have significant branching ratios into them, why isn't $N_{\text {eff }}$ of order $N$, the number of axions? We will investigate this question in this paper.

There have been a number of interesting prior studies on axionic dark radiation in string theory [17-27]. These papers consider examples which have very few light axions. Instead, our interest here is to the dependence of $N_{\text {eff }}$ on the number of light axions.

\section{The axiverse induced dark radiation problem}

We will illustrate the problem by beginning with a simple model and gradually considering more and more general (realistic) cases as we go on.

The simplest Lagrangian involving a modulus $(s)$, an axion $(t)$ and a gauge field strength $F_{\mu \nu}$ is arguably of the form:

$$
\frac{\mathcal{L}}{m_{p l}^{2}}=\frac{c}{s^{2}} \partial_{\mu} s \partial^{\mu} s+\frac{c}{s^{2}} \partial_{\mu} t \partial^{\mu} t+\tilde{c} s F_{\mu \nu} F^{\mu \nu}-\widetilde{m}^{2}\left(s-s_{o}\right)^{2}
$$

where $c$ and $\tilde{c}$ are constants. $s_{o}$ reflects that $s$ will have a non-zero vacuum expectation value. In our conventions, $s$ and $t$ are dimensionless and $m_{p l}$ is the Planck mass.

This form of the Lagrangian arises in supersymmetric string and $M$ theory models e.g. the universal axio-dilaton Lagrangian or the model independent axion/modulus multiplet in heterotic string compactifications [28]. From this Lagrangian we can canonically normalise 
the fields after setting $s$ to its vacuum value and compute the partial decay widths

$$
\Gamma(\hat{s} \rightarrow \hat{t} \hat{t})=\frac{1}{64 \pi c} \frac{m^{3}}{m_{p l}^{2}}
$$

and

$$
\Gamma(\hat{s} \rightarrow \gamma \gamma)=\frac{1}{64 \pi c} \frac{m^{3}}{m_{p l}^{2}}
$$

where $m=\frac{\widetilde{m}\langle s\rangle}{\sqrt{2 c}}$ is the physical moduli mass.

The contribution to dark radiation of axion from moduli decay can be calculated from $[17-19]$

$$
\Delta N_{\text {eff }}=\frac{43}{7} \frac{\Gamma_{\text {axions }}}{\Gamma_{\text {visible }}}\left(\frac{g^{*}}{g_{\text {reheat }}^{*}}\right)^{1 / 3}
$$

We can take the decay of moduli into two photons as a model for the decay of the moduli into Standard Model particles, so this calculation gives

$$
\Delta N_{\text {eff }} \sim O(1)
$$

since $\Delta N_{\text {eff }}$ is given by the ratio of the decay width of the modulus decay into axions versus Standard Model particles. This illustrates the fact that the moduli couple semi-universally to all particles (as one expects, since, after all they are extra dimensional gravitons).

In this paper we are interested in the case when there are a large number, $N$ of axion/moduli multiplets, $\left(t_{i}, s_{i}\right)$. The previous Lagrangian can then be generalised to

$$
\mathcal{L}=m_{p l}^{2} \sum_{i}\left(\frac{a_{i}}{s_{i}^{2}} \partial_{\mu} s_{i} \partial^{\mu} s_{i}+\frac{a_{i}}{s_{i}^{2}} \partial_{\mu} t_{i} \partial^{\mu} t_{i}+\tilde{a}_{i} s_{i} F_{\mu \nu} F^{\mu \nu}-\widetilde{m}_{i}^{2}\left(s_{i}^{2}-\left\langle s_{i}\right\rangle^{2}\right)\right)
$$

This Lagrangian arises from a supergravity theory containing $N$ chiral superfields with scalar components $z_{j}=t_{j}+i s_{j}$ with Kahler potential $K=-3 \ln V$ where $V=\Pi_{i} s_{i}^{a_{i}}$. This Kahler potential is a typical term which would arise in string/ $M$ theory compactifications. ${ }^{1}$ Let us now calculate $N_{\text {eff }} \equiv N_{S M}+\Delta N_{\text {eff }}$. To do this we need to evaluate the $N^{2}$ partial decay widths $\Gamma\left(\hat{s_{j}} \rightarrow \hat{t_{i}} \hat{t}_{i}\right)$ which can readily be calculated to be

$$
\Gamma\left(\hat{s_{j}} \rightarrow \hat{t_{i}} \hat{t_{i}}\right)=\frac{\delta_{i j}}{64 \pi} \frac{1}{a_{j}} \frac{m_{j}^{3}}{m_{p l}^{2}}
$$

where $m_{j}=\frac{\widetilde{m}_{j}\langle s\rangle}{\sqrt{2 a_{j}}}$. On the other hand we also calculate

$$
\Gamma\left(\hat{s_{j}} \rightarrow \gamma \gamma\right)=\frac{1}{64 \pi} \frac{1}{\left(\sum_{i} \widetilde{a}_{i}\left\langle s_{i}\right\rangle\right)^{2}} \frac{\widetilde{a}_{j}^{2}\left\langle s_{j}\right\rangle^{2}}{a_{j}} \frac{m_{j}^{3}}{m_{p l}^{2}}
$$

which results in

$$
\Delta N_{\mathrm{eff}}=\frac{\left(\sum_{i} \widetilde{a}_{i}\left\langle s_{i}\right\rangle\right)^{2}}{\widetilde{a}_{j}^{2}\left\langle s_{j}\right\rangle^{2}}=\frac{1}{(16 \pi \alpha)^{2} \widetilde{a}_{j}^{2}\left\langle s_{j}\right\rangle^{2}}
$$

where we used the fact that the sum which appears is related to the coupling constant of the gauge theory and have set the numerical factors in equation (2.4) to one for simplicity. Important points to note about this example are:

\footnotetext{
${ }^{1}$ In the next section we will study more concrete string/ $M$ theory examples.
} 
a) due to the diagonal mass and kinetic terms, a given modulus field $\hat{s}_{j}$ decays only into its axion partners;

b) the moduli with the smallest masses will decay last.

When the last (and lightest) modulus decays it substantially dilutes the energy density of particles produced from previous decays of heavier moduli. Hence, in computing $\Delta N_{\text {eff }}$ we are only interested in axions produced from the lightest moduli fields.

Now, in this particular case $\alpha$ is interpreted as the fine structure constant evaluated when the moduli decay takes place just before BBN, so $16 \pi \alpha$ is an order one number, independent of $N$. On the other hand, since $\frac{1}{16 \pi \alpha}$ is a sum of the $\mathrm{N}$ terms $\widetilde{a}_{j}\left\langle s_{j}\right\rangle$, if all $N$ terms contribute similar amounts to the sum, we would have $N_{\text {eff }} \sim N^{2}$ which is our first indication of the Axiverse induced dark radiation problem. In this particular, very special model, observational consistency requires that the value of $\alpha$ arises only from the modulus $s_{j}$ and hence that $\Delta N_{\text {eff }}$ is order one or smaller. Let us discuss more typical models.

In much more generality, the moduli dependent kinetic terms are not of the form $\frac{a_{i}}{s_{i}^{2}}$; rather they will be given by more complicated functions which are homogeneous of degree minus two. This is because the moduli Kahler potentials in string/ $M$ theory compactifications can be written as logarithms of homogeneous functions of fixed degrees, which implies that their second derivatives are homogeneous of said degree. Thus, one has a kinetic mixing matrix $K_{i j}$ whose entries are homogeneous of degree minus two. Before we discuss this most general case, we consider an intermediate, but instructive case: models in which the kinetic coefficients are diagonal, but arbitrary functions of degree minus two, $f_{i}$. This sort of example occurs when the Kahler potential is dominated by a single term, but which could depend on all the moduli. In this case we have, setting $m_{p l}=1$ :

$$
\mathcal{L}=f_{i}\left(\partial_{\mu} s_{i}\right)^{2}+f_{i}\left(\partial_{\mu} t_{i}\right)^{2}+\widetilde{a}_{i} s_{i} F_{\mu \nu}^{2}+\sum_{i} \widetilde{m}_{i}^{2} s_{i}^{2}
$$

Normalising the fields

$$
s_{i}=\frac{1}{\sqrt{2\left\langle f_{i}\right\rangle}} \hat{s}_{i}, \quad t_{i}=\frac{1}{\sqrt{2\left\langle f_{i}\right\rangle}} \hat{t}_{i}, \quad A_{\mu}=\frac{1}{2 \sqrt{\widetilde{a}_{i}\left\langle s_{i}\right\rangle}} \hat{A_{\mu}}
$$

gives the Lagrangian

$$
\mathcal{L}=\frac{1}{2}\left(\partial_{\mu} \hat{s}_{i}\right)^{2}+\frac{1}{2}\left(\partial_{\mu} \hat{t}_{i}\right)^{2}+\frac{1}{4} \hat{F}_{\mu \nu}^{2}+\frac{\left\langle\partial_{j} f_{i}\right\rangle}{2 \sqrt{2} \sqrt{\left\langle f_{j}\right\rangle}\left\langle f_{i}\right\rangle} \hat{s}_{j}\left(\partial_{\mu} \hat{t}_{i}\right)^{2}+\frac{\widetilde{a}_{j}}{4 \sqrt{2\left\langle f_{i}\right\rangle} \widetilde{a}_{i}\left\langle s_{i}\right\rangle} \hat{s}_{j} \hat{F}_{\mu \nu}^{2}
$$

This results in

$$
\begin{aligned}
\Gamma\left(\hat{s}_{j} \rightarrow \hat{t}_{i} \hat{t}_{i}\right) & =\frac{1}{256 \pi} \frac{1}{\left\langle f_{j}\right\rangle} \frac{1}{\left\langle f_{i}\right\rangle^{2}}\left\langle\partial_{j} f_{i}\right\rangle^{2} \frac{m_{j}^{3}}{m_{p l}^{2}} \\
\Gamma\left(\hat{s}_{j} \rightarrow \text { Axions }\right) & =\frac{1}{256 \pi}\left(\sum_{i=1}^{N} \frac{1}{\left\langle f_{j}\right\rangle} \frac{1}{\left\langle f_{i}\right\rangle^{2}}\left\langle\partial_{j} f_{i}\right\rangle^{2}\right) \frac{m_{j}^{3}}{m_{p l}^{2}} \\
\Gamma\left(\hat{s}_{j} \rightarrow \gamma \gamma\right) & =\frac{1}{64 \pi} \frac{1}{\left\langle f_{j}\right\rangle} \frac{\widetilde{a}_{j}^{2}}{\left(\sum_{i=1}^{N} \widetilde{a}_{i}\left\langle s_{i}\right\rangle\right)^{2}} \frac{m_{j}^{3}}{m_{p l}^{2}}
\end{aligned}
$$


where $m_{j}=\frac{\widetilde{m}_{j}}{\sqrt{2\left\langle f_{j}\right\rangle}}$. The key point here is the sum over $N$ terms in the second of the above equations. If the kinetic coefficient $f_{i}$ depends on $s_{j}$ then $s_{j}$ will be able to decay into $t_{i} t_{i}$ and, in the general case we will have $N$ such decays producing light axions, giving

$$
N_{\text {eff }} \propto N
$$

The fact that the decay width of the lightest moduli into axions is of order $N$ is independent of the moduli couplings to the hidden sector since it only depends on the number of fields.

It is also instructive to illustrate the $N$-dependence in simple examples as these demonstrate how the Axiverse induced dark radiation problem might be solved. In the first example we take all of the kinetic coefficients equal and to be given by

$$
f_{i}=\frac{1}{\sum_{k} s_{k}^{2}}=\frac{1}{s_{1}^{2}+\ldots+s_{N}^{2}} \equiv \frac{1}{S_{\mathrm{rms}}^{2}}
$$

The decay width of the j-th modulus to decay into the i-th axion is then

$$
\Gamma\left(\hat{s}_{j} \rightarrow \hat{t}_{i} \hat{t}_{i}\right)=\frac{1}{64 \pi} \frac{\left\langle s_{j}\right\rangle^{2}}{\left\langle S_{\mathrm{rms}}^{2}\right\rangle} \frac{m_{j}^{3}}{m_{p l}^{2}}
$$

which implies that the total decay width of the j-th modulus to decay into axions is a sum of $N$ terms which adds up to

$$
\Gamma\left(\hat{s}_{j} \rightarrow \text { axions }\right)=\frac{N}{64 \pi} \frac{\left\langle s_{j}\right\rangle^{2}}{\left\langle S_{\mathrm{rms}}^{2}\right\rangle} \frac{m_{j}^{3}}{m_{p l}^{2}}
$$

By comparison, the decay width into gauge bosons is

$$
\Gamma\left(\hat{s}_{j} \rightarrow \gamma \gamma\right)=\frac{1}{64 \pi} \frac{\widetilde{a}_{j}^{2}\left\langle S_{\mathrm{rms}}^{2}\right\rangle}{\left(\sum_{i=1}^{N} \widetilde{a}_{i}\left\langle s_{i}\right\rangle\right)^{2}} \frac{m_{j}^{3}}{m_{p l}^{2}}
$$

which leads to

$$
\Delta N_{\mathrm{eff}}\left(s_{j}\right)=N \frac{\left\langle s_{j}\right\rangle^{2}}{\left\langle S_{\mathrm{rms}}^{2}\right\rangle} \frac{\left(\sum_{i=1}^{N} \widetilde{a}_{i}\left\langle s_{i}\right\rangle\right)^{2}}{\widetilde{a}_{j}^{2}\left\langle S_{\mathrm{rms}}^{2}\right\rangle}=N \frac{\left\langle s_{j}\right\rangle^{2}}{\left\langle S_{\mathrm{rms}}^{2}\right\rangle} \frac{1}{(16 \pi \alpha)^{2} \widetilde{a}_{j}^{2}\left\langle S_{\mathrm{rms}}^{2}\right\rangle}
$$

Clearly, in this example, we can see that if the vev of $S_{\text {rms }}$ is sufficiently large in (11d units) then one can suppress the axion contribution to the dark radiation density.

Finally, let us discuss the most general case. The following Lagrangian:

$$
\mathcal{L}=\sum_{i=1}^{N} \sum_{j=1}^{N} C_{i j} U_{i k} s_{k} \partial_{\mu} t_{j} \partial^{\mu} t_{j}
$$

is the most general Lagrangian coupling moduli fields to axions with two derivatives of the axion fields. Here, $C_{i j}$ arises from diagonalising the Kahler metric $K_{i j}$ and $U_{i j}$, which 
we have ignored until now arises from diagonalising the moduli mass matrix. We supplement this Lagrangian with typical terms coupling the moduli to Standard Model and supersymmetric particles. The Lagrangian for moduli-gauge boson interactions is

$$
\mathcal{L}=\sum_{i=1}^{N} B_{i} U_{i k} s_{k} F_{\mu \nu} F^{\mu \nu}
$$

and the Lagrangian for moduli-scalar kinetic interactions is

$$
\mathcal{L}=\sum_{i=1}^{N} D_{i} U_{i k} s_{k} D_{\mu} f D^{\mu} f
$$

Dropping numerical factors, the decay width of $s_{k}$, into various channels is:

$$
\begin{aligned}
\Gamma_{\text {axions }} & =\sum_{j=1}^{N} \Gamma\left(s_{k} \rightarrow t_{j} t_{j}\right) \\
& =\sum_{j=1}^{N}\left(\sum_{i=1}^{N} C_{i j} U_{i k}\right)^{2} \frac{m_{s_{k}}^{3}}{M_{P L}^{2}} \\
\Gamma_{\text {gauge particles }} & =n_{G}\left(\sum_{i=1}^{N} B_{i} U_{i k}\right)^{2} \frac{m_{s_{k}}^{3}}{M_{P L}^{2}} \\
\Gamma_{\text {fermions/sfermions }} & =n_{f}\left(\sum_{i=1}^{N} D_{i} U_{i k}\right)^{2} \frac{m_{s_{k}}^{3}}{M_{P L}^{2}}
\end{aligned}
$$

where $n_{G}$ and $n_{f}$ are the numbers of gauge bosons and fermions respectively. Even though the most general model has so many parameters, one can see that we expect $N_{\text {eff }} \propto N$ :

$$
\left\langle\Delta N_{\text {eff }}\right\rangle \propto \frac{\Gamma_{\text {axions }}}{\Gamma_{\text {visible }}} \propto \frac{N\langle C\rangle^{2}}{n_{G}\langle B\rangle^{2}+n_{f}\langle D\rangle^{2}} \propto N
$$

This arises because we expect the mean values $C, B$ and $D$ to be comparable and that $\left(\sum_{i=1}^{N} U_{i k}\right)^{2}$ to be order one. This is borne out by explicit calculations, see e.g. [17-19, 2934]. In other words, since the moduli couplings to axions are comparable to their couplings to the Standard Model particles, the string/ $M$ theory axiverse is in serious tension with observed limits on the amount of dark radiation. In special examples with low numbers of axions, one can see that it is possible to generate acceptably small amounts of dark radiation assuming certain couplings are small enough, for example, [17-21, 24-26]. But in general, this will be difficult to avoid.

\section{$3 \quad$ String/ $M$ theory examples}

\subsection{Calabi-Yau compactifications}

In Calabi-Yau compactifications of superstring theories to four dimensions, The moduli and axion kinetic terms in the Lagrangian are derived from a function of the moduli fields called the Kahler potential, $K$, which, up to a coefficient is given by

$$
K=-a \ln V_{X}
$$


Here, $V_{X}$ is the volume of the Calabi-Yau manifold (as a function of the moduli). This is a sum of terms with coefficients given by the triple intersection numbers $d_{i j k}$. The coefficient $a$ takes different values, depending upon which string theory one is considering. In the heterotic and Type IIA compactifications, the volume is given as a function of the Kahler moduli $S_{i}$ as:

$$
V_{X}=\sum_{i=1}^{n} d_{i j k} S_{i} S_{j} S_{k}
$$

Clearly, in a completely generic case, with many non-zero entries in $d_{i j k}, V_{X}$ is a sum of many terms and $K_{i j}$ will not be diagonal. Hence, upon diagonalisation, when expanding around a particular vacuum state, the matrices $C_{i j}, U_{i j}$ and the coefficients $B_{i}$ and $D_{i}$ will be quite general and we expect $N_{\text {eff }} \propto N$.

In the LARGE volume scenario of [33, 34], there is a modulus field with a vev much larger than that of the other moduli. In this case, the volume functional of the Calabi-Yau threefold is approximated by

$$
\begin{aligned}
& V=s_{1}^{3 / 2}-s_{2}^{3 / 2}-\ldots-s_{N}^{3 / 2} \\
& K=-2 \ln V
\end{aligned}
$$

In the limit where the $s_{1}$ vev is larger than the other vevs, $s_{1} \gg s_{i}$, the diagonalised Kahler metric is approximately

$$
\begin{aligned}
f_{1} & =K_{11} \approx \frac{3}{4 s_{1}^{2}}, & f_{i} & =K_{i i} \approx \frac{3}{8 s_{1}^{3 / 2} s_{i}^{1 / 2}} \\
\partial_{1} f_{1} & =-\frac{3}{2 s_{1}^{3}}, \quad \partial_{i} f_{1}=0, & \partial_{1} f_{i} & =-\frac{9}{16 s_{1}^{5 / 2} s_{i}^{1 / 2}},
\end{aligned}
$$

For $s_{1}$, it turns out that $\Delta N_{\text {eff }} \propto N$. This can be seen as follows. The decay widths to axions are

$$
\begin{aligned}
\Gamma\left(\hat{s}_{1} \rightarrow \hat{t}_{1} \hat{t}_{1}\right) & =\frac{1}{256 \pi}\left(\frac{16}{3}\right) \frac{m^{3}}{m_{p l}^{2}} \\
\Gamma\left(\hat{s}_{1} \rightarrow \hat{t}_{i \neq 1} \hat{t}_{i \neq 1}\right) & =\frac{3}{256 \pi} \frac{m^{3}}{m_{p l}^{2}} \\
\Gamma\left(\hat{s}_{1} \rightarrow \text { axions }\right) & =\frac{1}{256 \pi}\left(\frac{16}{3}+3(N-1)\right) \frac{m^{3}}{m_{p l}^{2}}
\end{aligned}
$$

whilst the gauge boson channel gives

$$
\Gamma\left(\hat{s}_{1} \rightarrow \gamma \gamma\right)=\frac{1}{48 \pi} \frac{\widetilde{a}_{j}^{2}\left\langle s_{1}^{2}\right\rangle}{\left(\sum_{i=1}^{N} \widetilde{a}_{i}\left\langle s_{i}\right\rangle\right)^{2}} \frac{m^{3}}{m_{p l}^{2}}
$$

resulting in a dark radiation contribution of

$$
\Delta N_{\mathrm{eff}}\left(s_{1}\right)=\left(1+\frac{9}{16}(N-1)\right) \frac{\left(\sum_{i=1}^{N} \widetilde{a}_{i}\left\langle s_{i}\right\rangle\right)^{2}}{\widetilde{a}_{1}^{2}\left\langle s_{1}^{2}\right\rangle}
$$


This is interesting, because in LARGE volume models, the vev $s_{1}$ is expected to be much larger than the other vevs, hence one expects a suppression of $\Delta N_{\text {eff }}$ in this case, following our discussion in section two. Furthermore, $s_{1}$ is typically the lightest modulus in this scenario [34].

For completeness, for $s_{j \neq 1}$, the dark radiation density doesn't depend on $N$ :

$$
\begin{aligned}
\Gamma\left(\hat{s}_{j \neq 1} \rightarrow \hat{t}_{j \neq 1} \hat{t}_{j \neq 1}\right) & =\frac{1}{128 \pi} \frac{\left\langle s_{1}\right\rangle^{3 / 2}}{\left\langle s_{j}\right\rangle^{3 / 2}} \frac{m^{3}}{m_{p l}^{2}} \\
\Gamma\left(\hat{s}_{j \neq 1} \rightarrow \hat{t}_{j \neq i} \hat{t}_{j \neq i}\right) & =0 \\
\Gamma\left(\hat{s}_{j \neq 1} \rightarrow \text { axions }\right) & =\frac{1}{128 \pi} \frac{\left\langle s_{1}\right\rangle^{3 / 2}}{\left\langle s_{j}\right\rangle^{3 / 2}} \frac{m^{3}}{m_{p l}^{2}}
\end{aligned}
$$

gauge boson channel is

$$
\Gamma\left(\hat{s}_{j \neq 1} \rightarrow \gamma \gamma\right)=\frac{1}{24 \pi} \frac{\widetilde{a}_{j}^{2}\left\langle s_{j}^{1 / 2}\right\rangle\left\langle s_{1}^{3 / 2}\right\rangle}{\left(\sum_{i=1}^{N} \widetilde{a}_{i}\left\langle s_{i}\right\rangle\right)^{2}} \frac{m^{3}}{m_{p l}^{2}}
$$

So the total dark radiation density is proportional to

$$
\Delta N_{\text {eff }}\left(s_{j \neq 1}\right)=\frac{3}{16} \frac{\left(\sum_{i=1}^{N} \widetilde{a}_{i}\left\langle s_{i}\right\rangle\right)^{2}}{\widetilde{a}_{j}^{2}\left\langle s_{j}^{2}\right\rangle}
$$

\subsection{Diagonal Kahler metrics}

Clearly, from the above discussions, one can suppress dark radiation from moduli decays when the Kahler metric for the moduli fields is approximately diagonal. This will be the case when the Volume function is dominated by just one term only.

$$
V_{X}=\prod_{i=1}^{N} S_{i}^{a_{i}}, \quad K=-3 \sum_{i=1}^{N} a_{i} \ln S_{i}
$$

where $a_{i}$ are microscopic parameters whose sum is a constant determined by the geometry of the extra dimensions. This is unity for the Calabi-Yau case and $\frac{7}{3}$ for $G_{2}$-manifolds. To calculate decay width, let us translate the above internal manifold into condition on decay width coefficients particularly $C_{i j}$. It can be shown that (see appendix A)

$$
\begin{aligned}
C_{i j} & =\frac{1}{\sqrt{K_{i i}^{D}}} \frac{\partial \ln K_{i i}^{D}}{\partial s_{j}} \\
B_{i} & =\frac{\alpha}{\sqrt{K_{i i}^{D}}} N_{i} \\
D_{i} & =\frac{1}{\sqrt{K_{i i}^{D}}} \frac{\partial \ln K_{\alpha \alpha}^{D}}{\partial s_{i}}
\end{aligned}
$$

where $K_{i j}^{D}$ is the diagonal Kahler metric. From (3.17), It is trivial to show that the coefficients are also diagonal:

$$
C_{i j}=C_{i} \delta_{i j}
$$


Following the previous analysis, this simple relation implies that $\Delta N_{\text {eff }}$ becomes independent of $N$ on average.

$$
\left\langle\Delta N_{\mathrm{eff}}\right\rangle \propto \frac{\langle C\rangle^{2}}{n_{G}\langle B\rangle^{2}+n_{f}\langle D\rangle^{2}}
$$

where the orthogonality of rotation matrix, $\left(\sum_{i=1}^{N} U_{i k}^{2}\right)=1$, has been used.

The physical reason for this behaviour is that this particular volume form forces each modulus to decay only into its axionic partner. If we assume further that this basis is already physical, i.e. there is no further mixing between moduli or axions, it becomes clear that dark radiation, regardless of $\mathrm{N}$, consists of only one species of axion which is the partner of the last modulus to decay.

The moduli mixing matrix can also play a role in suppressing dark radiation. Again, though non-generic, this occurs when there is a relation between the moduli mass matrix and the eigenvalues of the Kahler metric:

$$
\sqrt{K_{i i}^{D}} \propto U_{i j}
$$

The above relation is equivalent to

$$
\frac{1}{C_{i}} \propto U_{i k}, \frac{1}{B_{i}} \propto U_{i k}, \frac{1}{D_{i}} \propto U_{i k}
$$

In this case, the correlation becomes

$$
\left\langle\Delta N_{\mathrm{eff}}\right\rangle \propto \frac{N\langle C\rangle^{2}}{n_{G} N^{2}\langle B\rangle^{2}+n_{f} N^{2}\langle D\rangle^{2}} \propto \frac{1}{N}
$$

Therefore, under these very special circumstances, dark radiation can actually be suppressed by the number of axions on average. This counter-intuitive result is merely the effect of increasing $\mathrm{N}$-dependence of the moduli to visible sector couplings so that dark radiation is dominated by standard model radiation (neutrinos). Most likely, this is merely a curious observation rather than a realistic case.

\subsubsection{Mass matrix in $G_{2}$ compactified M-theory}

In this subsection, we put together some of these results in a concrete setting where the moduli mass matrix is known, namely $G_{2}$-compactified $M$ theory. Again, we are assuming that $K$ is dominated by a single term:

$$
K=-3 \ln \left(\prod_{i=1}^{N} s_{i}^{a_{i}}\right)
$$

where $\sum_{i=1}^{N} a_{i}=\frac{7}{3}$. From above, $N_{\text {eff }}$ becomes independent of the number of axions in this model. However, regardless of this advantage, one could easily find that the typical value of $N_{\text {eff }}$, although independent of $N$, is actually too large in practice e.g. $\Delta N_{\text {eff }} \sim 10$. We would therefore like to investigate the possibility of further suppressing dark radiation in this setup. 
We briefly recall some details of moduli stabilisation. It has been shown in [29] that with a hidden sector with two gauge groups where first group is SQCD with 1 flavour of quarks and second group is pure glue sQCD leads to dS vacua. The superpotential is written as

$$
W=A_{1} \phi^{a} e^{i b_{1} \sum_{i}^{N} N_{i} S_{i}}+A_{2} e^{i b_{2} \sum_{i}^{N} N_{i} S_{i}}
$$

where $\phi$ is the meson superfield in the hidden sector. With the superpotential and Kahler potential being specified, it is straightforward yet tedious to work out the mass mixing matrix resulting from moduli stabilisation [30, 32].

$$
\begin{array}{rlrl}
U_{k j} & =\sqrt{\frac{a_{j+1}}{\left(\sum_{i=1}^{j} a_{i}\right)\left(\sum_{i=1}^{j+1} a_{i}\right)}} \sqrt{a_{k}}, & & k \leq j \\
U_{k j} & =-\sqrt{\frac{\sum_{i=1}^{j} a_{i}}{\sum_{i=1}^{j+1} a_{i}}}, & k=j+1 \\
U_{k N} & =\sqrt{\frac{3 a_{k}}{7}} &
\end{array}
$$

where $i=1 \ldots N-1$ are the degenerate light moduli and $i=N$ is heavy modulus. Notice that except $k=j+1, U_{k j} \propto \sqrt{a_{k}} \propto \sqrt{K_{k}}$. Therefore, we the element $U_{j+1, j}$ will be suppressed if it turned out that:

$$
\sum_{i=1}^{j} a_{i} \ll a_{j+1}
$$

As a result, one would expect $\frac{1}{N}$ suppression on dark radiation under this condition.

The modulus decay width can be calculated from [30]

$$
\begin{aligned}
\Gamma_{X_{j}} & =D_{X_{j}} \frac{m_{X_{j}}^{3}}{M_{P l}^{2}} \\
D_{X_{j}} & =\alpha\left(\sum_{k=1}^{N} \frac{U_{k j}^{2}}{a_{k}}\right)+\beta\left(\sum_{k=1}^{N} \frac{U_{k j}}{\sqrt{a_{k}}}\right)^{2}
\end{aligned}
$$

where $\alpha$ and $\beta$ are index-independent parameters dependent on the microscopic details of the $G_{2}$ manifold. The first term represents the decay width into axions where the latter represents decay width into visible particles. From (3.26) and (3.28) it is trivial to see that total decay width of $j^{\text {th }}$ modulus and corresponding dark radiation are controlled by

$$
\begin{aligned}
\Gamma_{j} & \propto \frac{\sum_{i=1}^{j} a_{i}}{a_{j+1}} \\
\Delta N_{\mathrm{eff}}\left(X_{j}\right) & \propto \frac{j a_{j+1}^{2}+\left(\sum_{i=1}^{j} a_{i}\right)^{2}}{\left(j a_{j+1}-\sum_{i=1}^{j} a_{i}\right)^{2}}
\end{aligned}
$$

Applying (3.27), we clearly see that $\Delta N_{\text {eff }}\left(X_{j}\right) \propto \frac{1}{j}$ and $\Gamma_{j}$ becomes smallest. This is essential to the model because it guarantees that the last decay modulus exhibits $\frac{1}{N}$ behaviour. For practical purpose, only $j=N-1$ in condition (3.27) will be assumed. 




(a)

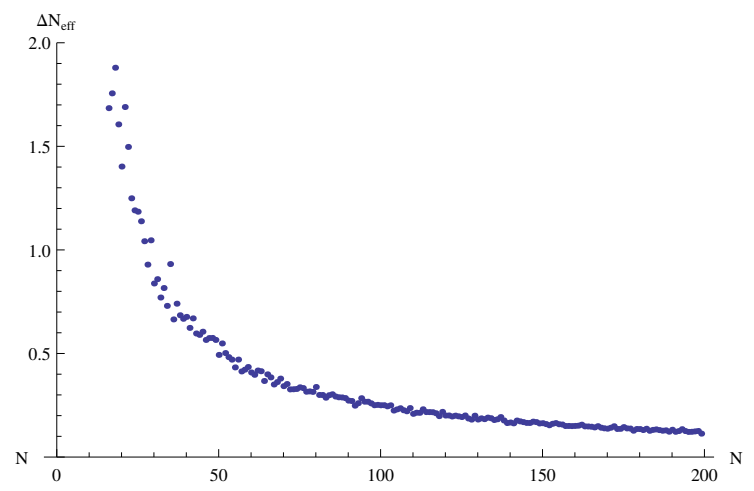

(b)

Figure 1. Left: result from geometric sequence configurations showing $\Delta N_{\text {eff }}$ as a function of $N$, where $r=2$. Right: result from double moduli dominated configurations showing $\Delta N_{\text {eff }}$ as a function of $N$, where $\epsilon N=0.1$.

Next, we will explicitly show correlations between the number of axions and $N_{\text {eff }}$. Instead of scanning the $N$ parameters $a_{i}$ space, we will give systematic examples of simple configurations of the $a_{i}$ which work: the first example is when $n$ of the $a_{i}$ are large and the rest small:

$$
a_{i}=\{\underbrace{\epsilon \bar{a}, \ldots, \epsilon \bar{a}}_{N-n}, \underbrace{\frac{7}{3 n}-\frac{\epsilon \bar{a}(N-n)}{n}, \ldots, \frac{7}{3 n}-\frac{\epsilon \bar{a}(N-n)}{n}}_{n}\}
$$

The second is a "geometric sequence" of $a_{i}$ 's,

$$
a=\left\{a_{0}, a_{0}, a_{0} r, a_{0} r^{2}, \ldots, a_{0} r^{N-2}\right\}
$$

Though these can be viewed as toy models at best, they both illustrate that, in principle, the amount of dark radiation can actually decrease as one increases the number of axions. This is illustrated in the two figures.

\section{Conclusions and outlook}

$\Delta N_{\text {eff }}$ is a very powerful probe of light degrees of freedom in the hidden sector and, somewhat surprisingly, has been constrained to be quite small, consistent with zero. The Axiverse induced Dark Radiation Problem arises from the plethora of light degrees of freedom that can be present in string/ $M$ theory compactifications to four dimensions. Though we focused on the axions, similar conclusions can be drawn from hidden photons and other light particles in the hidden sector. We pointed out several possible mechanisms via which this problem could be avoided: a) a relatively large modulus vev as in the LARGE volume scenario; b) alignment between the axion kinetic and mass mixing matrices so that the last modulus to decay does so predominantly into its axionic partner. It would be very interesting to explore these mechanisms in more detail in various specific models. One potential problem with the large vev solution in practice is that the large vev corresponds 


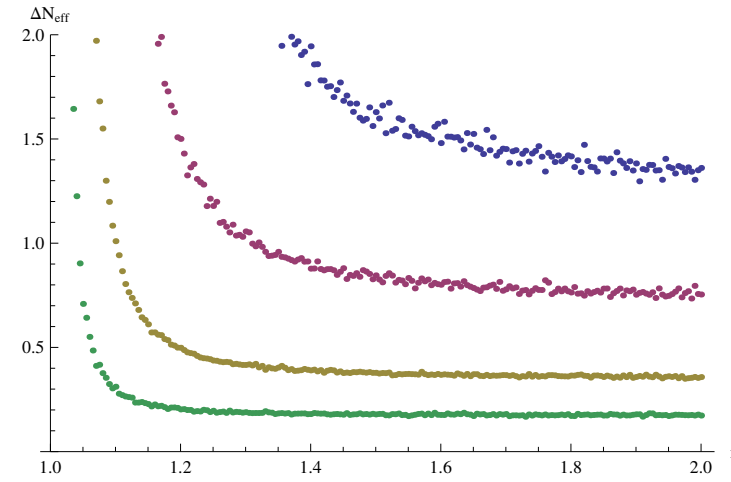

(a)

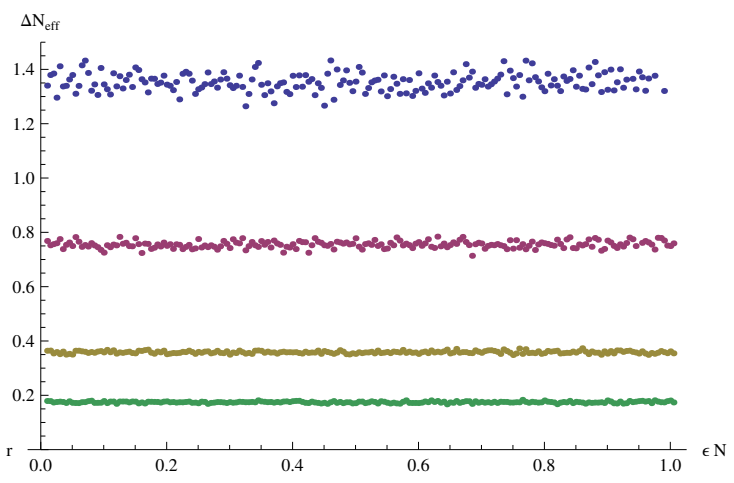

(b)

Figure 2. Left: result from geometric sequence configurations showing $\Delta N_{\text {eff }}$ as a function of $r$, where points in blue, red, yellow, green are $N=30,50,100,200$ respectively. Right: result from double moduli dominated configurations showing $\Delta N_{\text {eff }}$ as a function of $\epsilon N$, where points in blue, red, yellow, green are $N=30,50,100,200$ respectively.

to a weak Standard Model coupling. In general, it might be difficult to make the vev large enough without making the Standard Model coupling too small.

\section{Acknowledgments}

The work of BSA is supported by the U.K. STFC via the research grant ST/J002798/1. CP is supported by the KCL NMS graduate school and ICTP Trieste.

\section{A Decay coefficients}

We show detail analysis for coefficients $C_{i j}, B_{i}$ and $D_{i}$ in this section. The kinetics terms for moduli and axions are controlled by Kahler metric as following

$$
\mathcal{L}=\frac{1}{2} K_{i j} \partial^{\mu} s^{i} \partial_{\mu} s^{j}+\frac{1}{2} K_{i j} \partial^{\mu} t^{i} \partial_{\mu} t^{j}
$$

After canonically normalisation of moduli and axions, we can expand Kahler metric as a function of moduli field. After taking moduli mixing into account, the result for interaction Lagrangian is

$$
\mathcal{L}_{\tilde{s}_{k} \tilde{t}_{i} \tilde{t}_{i}}=\frac{1}{2} \frac{\sum_{j=1}^{N} \frac{\partial K_{i i}^{D}}{\partial s_{j}} U_{j k}}{\left(K_{i i}^{D}\right)^{3 / 2}} \tilde{s}_{k} \partial^{\mu} \tilde{t}^{i} \partial_{\mu} \tilde{t}^{j}
$$

where $K^{D}$ is Kahler metric after diagonalisation. $\tilde{s}, \tilde{t}$ are canonically normalised fields after mixing. Straightforwardly, one can derive decay width into axions as

$$
\Gamma_{\text {axions }}=\frac{1}{32 \pi} \sum_{i=1}^{N}\left(\sum_{j=1}^{N} \frac{1}{\sqrt{K_{i i}^{D}}} \frac{\partial \ln K_{i i}^{D}}{\partial s_{j}} U_{j k}\right)^{2} \frac{m_{X_{k}}^{3}}{M_{p l}^{2}}
$$


For gauge sector, the Lagrangian takes the form

$$
\mathcal{L}=-\frac{1}{4}\left(\sum_{i=1}^{N} N_{i} z_{i}\right) F_{\mu \nu} F^{\mu \nu}
$$

After canonically normalisation of moduli and gauge fields and mixing between moduli, we get the interaction terms between moduli and gauge fields.

$$
\mathcal{L}=\frac{1}{4} \frac{1}{\left(\sum_{i=1}^{N} N_{i}\left\langle s_{i}\right\rangle\right)} \sum_{i=1}^{N} \frac{N_{i} U_{i k}}{\sqrt{K_{i i}^{D}}} \tilde{s}_{k} F_{\mu \nu} F^{\mu \nu}
$$

The moduli decay width into gauge bosons/gauginos is given by

$$
\Gamma_{\text {gauge }}=\frac{N_{G}}{32 \pi}\left(\sum_{i=1}^{N} \frac{\alpha}{\sqrt{K_{i i}^{D}}} N_{i} U_{i k}\right)^{2} \frac{m_{X_{k}}^{3}}{M_{p l}^{2}}
$$

For matter sector, the interaction terms can be found from

$$
\mathcal{L}=K_{\alpha \beta} D_{\mu} f^{\alpha} D^{\mu} f^{\beta}+K_{\alpha \beta} \tilde{f}^{\alpha} \not D \tilde{f}^{\beta}
$$

Then, after normalisation of moduli field and fermions/sfermions fields, the interaction terms become

$$
\mathcal{L}=\sum_{i=1}^{N} \frac{1}{\sqrt{K_{i i}^{D}}} \frac{\partial \ln K_{\alpha \alpha}^{D}}{\partial s_{i}} U_{i k} \tilde{s}_{k}\left(D_{\mu} f^{\alpha} D^{\mu} f^{\alpha}+\tilde{f}^{\alpha} \not D \tilde{f}^{\alpha}\right)
$$

Therefore, the decay width into fermion can be written as

$$
\Gamma_{\text {fermion }} \propto\left(\sum_{i=1}^{N} \frac{1}{\sqrt{K_{i i}^{D}}} \frac{\partial \ln K_{\alpha \alpha}^{D}}{\partial s_{i}} U_{i k}\right)^{2} \frac{m_{X_{k}}^{3}}{M_{p l}^{2}}
$$

Comparing to previous section, we get

$$
\begin{aligned}
C_{i j} & =\frac{1}{\sqrt{K_{i i}^{D}}} \frac{\partial \ln K_{i i}^{D}}{\partial s_{j}} \\
B_{i} & =\frac{\alpha}{\sqrt{K_{i i}^{D}}} N_{i} \\
D_{i} & =\frac{1}{\sqrt{K_{i i}^{D}}} \frac{\partial \ln K_{\alpha \alpha}^{D}}{\partial s_{i}}
\end{aligned}
$$

Open Access. This article is distributed under the terms of the Creative Commons Attribution License (CC-BY 4.0), which permits any use, distribution and reproduction in any medium, provided the original author(s) and source are credited. 


\section{References}

[1] S. Deser and E. Witten, Dynamical properties of antisymmetric tensor fields, Nucl. Phys. B 178 (1981) 491 [INSPIRE].

[2] P. Svrček and E. Witten, Axions in string theory, JHEP 06 (2006) 051 [hep-th/0605206] [INSPIRE].

[3] A. Arvanitaki, S. Dimopoulos, S. Dubovsky, N. Kaloper and J. March-Russell, String axiverse, Phys. Rev. D 81 (2010) 123530 [arXiv:0905.4720] [INSPIRE].

[4] J. Preskill, M.B. Wise and F. Wilczek, Cosmology of the invisible axion, Phys. Lett. B 120 (1983) 127 [INSPIRE].

[5] L.F. Abbott and P. Sikivie, A cosmological bound on the invisible axion, Phys. Lett. B 120 (1983) 133 [INSPIRE].

[6] M. Dine and W. Fischler, The not so harmless axion, Phys. Lett. B 120 (1983) 137 [INSPIRE].

[7] T. Banks, D.B. Kaplan and A.E. Nelson, Cosmological implications of dynamical supersymmetry breaking, Phys. Rev. D 49 (1994) 779 [hep-ph/9308292] [INSPIRE].

[8] B. de Carlos, J.A. Casas, F. Quevedo and E. Roulet, Model independent properties and cosmological implications of the dilaton and moduli sectors of $4 D$ strings, Phys. Lett. B 318 (1993) 447 [hep-ph/9308325] [INSPIRE].

[9] G. Lazarides, R.K. Schaefer, D. Seckel and Q. Shafi, Dilution of cosmological axions by entropy production, Nucl. Phys. B 346 (1990) 193 [INSPIRE].

[10] P. Fox, A. Pierce and S.D. Thomas, Probing a QCD string axion with precision cosmological measurements, hep-th/0409059 [INSPIRE].

[11] J. Kaplan, Dark matter generation and split supersymmetry, JHEP 10 (2006) 065 [hep-ph/0601262] [INSPIRE].

[12] B.S. Acharya, K. Bobkov and P. Kumar, An M-theory solution to the strong CP problem and constraints on the axiverse, JHEP 11 (2010) 105 [arXiv: 1004.5138] [INSPIRE].

[13] WMAP collaboration, G. Hinshaw et al., Nine-year Wilkinson Microwave Anisotropy Probe (WMAP) observations: cosmological parameter results, Astrophys. J. Suppl. 208 (2013) 19 [arXiv: 1212.5226] [INSPIRE].

[14] Z. Hou et al., Constraints on cosmology from the cosmic microwave background power spectrum of the $2500 \mathrm{deg}^{2}$ SPT-SZ survey, Astrophys. J. 782 (2014) 74 [arXiv:1212.6267] [INSPIRE].

[15] Atacama Cosmology Telescope collaboration, J.L. Sievers et al., The Atacama Cosmology Telescope: cosmological parameters from three seasons of data, JCAP 10 (2013) 060 [arXiv: 1301.0824] [INSPIRE].

[16] Planck collaboration, P.A.R. Ade et al., Planck 2015 results. XIII. Cosmological parameters, arXiv:1502.01589 [INSPIRE].

[17] M. Cicoli, J.P. Conlon and F. Quevedo, Dark radiation in LARGE volume models, Phys. Rev. D 87 (2013) 043520 [arXiv:1208.3562] [INSPIRE].

[18] T. Higaki and F. Takahashi, Dark radiation and dark matter in large volume compactifications, JHEP 11 (2012) 125 [arXiv:1208.3563] [INSPIRE]. 
[19] T. Higaki, K. Nakayama and F. Takahashi, Moduli-induced axion problem, JHEP 07 (2013) 005 [arXiv: 1304.7987] [INSPIRE].

[20] J.P. Conlon and M.C.D. Marsh, The cosmophenomenology of axionic dark radiation, JHEP 10 (2013) 214 [arXiv: 1304.1804] [INSPIRE].

[21] S. Angus, J.P. Conlon, U. Haisch and A.J. Powell, Loop corrections to $\Delta N_{\text {eff }}$ in large volume models, JHEP 12 (2013) 061 [arXiv:1305.4128] [INSPIRE].

[22] M. Cicoli, Axion-like particles from string compactifications, arXiv:1309.6988 [INSPIRE].

[23] M. Cicoli, J.P. Conlon, M.C.D. Marsh and M. Rummel, $3.55 \mathrm{keV}$ photon line and its morphology from a $3.55 \mathrm{keV}$ axionlike particle line, Phys. Rev. D 90 (2014) 023540 [arXiv: 1403.2370] [INSPIRE].

[24] S. Angus, Dark radiation in anisotropic LARGE volume compactifications, JHEP 10 (2014) 184 [arXiv: 1403.6473] [INSPIRE].

[25] A. Hebecker, P. Mangat, F. Rompineve and L.T. Witkowski, Dark radiation predictions from general large volume scenarios, JHEP 09 (2014) 140 [arXiv: 1403.6810] [INSPIRE].

[26] M. Cicoli and F. Muia, General analysis of dark radiation in sequestered string models, JHEP 12 (2015) 152 [arXiv: 1511. 05447] [INSPIRE].

[27] D.J.E. Marsh, Axion cosmology, arXiv:1510.07633 [INSPIRE].

[28] M.B. Green, J.H. Schwarz and E. Witten, Superstring theory. Vol. 2: loop amplitudes, anomalies and phenomenology, Cambridge Univ. Pr., Cambridge U.K. (1988) [INSPIRE].

[29] B.S. Acharya, K. Bobkov, G.L. Kane, P. Kumar and J. Shao, Explaining the electroweak scale and stabilizing moduli in M-theory, Phys. Rev. D 76 (2007) 126010 [hep-th/0701034] [INSPIRE].

[30] B.S. Acharya, P. Kumar, K. Bobkov, G. Kane, J. Shao and S. Watson, Non-thermal dark matter and the moduli problem in string frameworks, JHEP 06 (2008) 064 [arXiv: 0804.0863] [INSPIRE].

[31] B.S. Acharya, K. Bobkov, G.L. Kane, J. Shao and P. Kumar, The $G_{2}-M S S M$ : an M-theory motivated model of particle physics, Phys. Rev. D 78 (2008) 065038 [arXiv:0801.0478] [INSPIRE].

[32] B.S. Acharya and K. Bobkov, Kähler independence of the $G_{2}$-MSSM, JHEP 09 (2010) 001 [arXiv:0810.3285] [INSPIRE].

[33] V. Balasubramanian, P. Berglund, J.P. Conlon and F. Quevedo, Systematics of moduli stabilisation in Calabi-Yau flux compactifications, JHEP 03 (2005) 007 [hep-th/0502058] [INSPIRE].

[34] J.P. Conlon, F. Quevedo and K. Suruliz, Large-volume flux compactifications: moduli spectrum and D3/D7 soft supersymmetry breaking, JHEP 08 (2005) 007 [hep-th/0505076] [INSPIRE]. 